\title{
CRESCIMENTO DE MUDAS DE PUPUNHEIRA (Bactris gasipaes) TRANSPLANTADAS EM DIFERENTES ESTÁDIOS DE PLÂNTULA, SUBSTRATOS E VOLUME DE SUBSTRATO
}

\author{
Kaoru YUYAMA ${ }^{1}$, Sílvia M.S. MESQUITA ${ }^{2}$
}

\begin{abstract}
RESUMO - A Comissão Estadual de Sementes e Mudas do Estado do Amazonas estabeleceu normas e padrões para a produção de mudas fiscalizadas, mas esses padrões ainda são vagos para a pupunheira (Bactris gasipaes Kunth, Palmae). Visando melhorar esse padrão avaliou-se o desenvolvimento de mudas transplantadas em diferentes estádios de plântula (chifrinhos, uma folha e duas folhas), substrato (sem e com esterco de galinha: $3: 1 \mathrm{v}: \mathrm{v}$ de solo superficial: esterco) e volume de substrato $(0,5,1$ e $2 \mathrm{~kg})$, em Manaus, AM. Foram utilizadas sementes de plantas inermes de Yurimaguas, Peru, colhidas em março de 1997. Adotou-se o delineamento de blocos casualizados, em esquema fatorial $2 \times 3 \times 3$, com quatro repetições. As plântulas em substrato com esterco tiveram maior crescimento em altura $(19 \mathrm{~cm})$ em relação às sem esterco $(8 \mathrm{~cm})$ aos 6 meses, e as plântulas transplantadas no estádio de uma folha tiveram maior crescimento $(15 \mathrm{~cm})$, seguida de chifrinho $(14 \mathrm{~cm})$ e de duas folhas $(12 \mathrm{~cm})$. Os sacos com capacidade para $1 \mathrm{~kg}$ e 2 $\mathrm{kg}$ não diferiram no crescimento das plantas (altura 14,5 e 15,0 cm, respectivamente), superando o de $0,5 \mathrm{~kg}(11 \mathrm{~cm})$. Portanto, para a produção de mudas de boa qualidade, as plântulas de pupunheira devem ser transplantadas no estádio de uma folha aberta, em sacos de um kg contendo substrato com esterco.
\end{abstract}

Palavras-chave: adubação orgânica, viveiro, produção de mudas, padrão de qualidade,

Growth of Peach Palm (Bactris gasipaes) Seedlings Transplanted in Different Seedling Stages, Substrates and Substrate Volumes

ABSTRACT - The Amazonas State Commission of Seeds and Seedlings established norms and standards for the production of certified seedlings, but for peach palm seedlings they are poorly defined due to lack of specific studies. The objective of this study was to investigate the development of seedlings transplanted at different seedling stages (before appearance of leaves, one leaf and two leaves), substrate type (surface soil and surface soil + chicken manure $3: 1 \mathrm{v}: \mathrm{v}$ ), and substrate volume $(0.5,1$ and $2 \mathrm{~kg}$ bags), in Manaus, AM, Brazil. The seeds were from spineless Yurimaguas, Peru, plants, collected in March 1997. The experimental design was a randomized blocks, with a $2 \times 3 \times 3$ factorial, with four replications. The seedlings fertilized with chicken manure presented better growth in height $(19 \mathrm{~cm})$ than those in surface soil $(8 \mathrm{~cm})$ at six months. Seedlings with one leaf had greater development $(15 \mathrm{~cm})$, followed by seedlings without leaves $(14 \mathrm{~cm})$ or with two leaves $(12 \mathrm{~cm})$. Seedlings in bags with one $(14.5 \mathrm{~cm})$ and two $(15 \mathrm{~cm}) \mathrm{kg}$ capacities presented better growth than these in bags with $0.5 \mathrm{~kg}(11 \mathrm{~cm})$. Therefore, peach palm seedlings should be transplanted at the one leaf seedling stage into bags with one $\mathrm{kg}$ of soil plus chicken manure.

Key-words: organic fertilizer, nursery, seedling production, seedling standards, pejibaye.

A pupunheira (Bactris gasipaes Kunth, Palmae) é uma planta típica da
Amazônia que produz frutos de alto valor nutritivo e sabor agradável.

\footnotetext{
'Instituto Nacional de Pesquisas da Amazônia, Caixa Postal 478, 69.011-970 Manaus, AM, Brasil. ${ }^{2}$ Bolsista CNPq-PIBIC/INPA.
} 
Alguns pesquisadores consideram 0 fruto da pupunheira um substituto em potencial para parte das proteínas animais consumidas pela população da Amazônia (Clement \& Mora Urpí, 1987). Os frutos possuem potencial econômico para farinha, óleo e ração animal, e a planta para produção de palmito (Mora Urpi et al., 1982; Clement \& Mora Urpí, 1987) e para confecção de artesanato e moveis do estipe. Atualmente, vem despertando o interesse de empresários e agricultores locais, assim como em outras regiões do Brasil, devido as amplas possibilidades como produtora de palmito.

As pesquisas com a pupunheira para produção de palmito iniciaram em 1965 no Centro Agronômico Tropical de Pesquisa e Ensino (CATIE), em Turrialba, Costa Rica (Clement \& Mora Urpi, 1987). Hoje, já existem informações sobre sistemas de produção de pupunheira (densidade de plantio, adubação, tratos culturais, época de extração do palmito, época da colheita de frutos; Villachica, 1996), mas as informações são escassas no que concerne a produção de mudas. As normas e padrões para a produção de mudas fiscalizadas de pupunheira, elaboradas pela Comissão Estadual de Sementes e Mudas (CESM, 1996), ainda são vagas devido à falta de estudos especificos. Questões importantes sobre adubação, tamanho do saco plástico (volume de substrato) e estádio ideal de desenvolvimento da plântula para repicagem ainda não estão devidamente elucidados. Um maior tamanho de saco (com maior quantidade de substrato) pode proporcionar melhor desenvolvimento das mudas (Villachica, 1996), mas aumenta o custo do enchimento dos sacos, bem como do transporte das mudas do viveiro para o local de plantio. Para os produtores de mudas é vantajoso produzir mudas em curto espaço de tempo e em sacos com menor quantidade de substrato, pois reduz custos. Desta forma, necessitase uma recomendação mais prática. Este trabalho teve como objetivo avaliar o crescimento de mudas de pupunheiras transplantadas em diferentes estádios, tamanho de recipiente (com diferentes volumes de substrato) e adubação.

As sementes de pupunheira utilizadas foram coletadas em março de 1997 de plantas inermes de Yurimaguas, Peru. O experimento foi conduzido no viveiro do Instituto Nacional de Pesquisas da Amazônia, Manaus, AM. Utilizou-se o delineamento de blocos casualizados, em esquema fatorial $2 \times 3 \times 3$, com quatro repetições de 10 plântulas, e os seguintes tratamentos: dois tipos de substrato (sem esterco de galinha; 3:1 $\mathrm{v}: \mathrm{v}$ de solo superficial : esterco), três estádios de plântulas (chifrinho; uma folha; duas folhas) e três tamanhos de sacos de polietileno preto $(12 \times 25 \mathrm{~cm}$ $-0,5 \mathrm{~kg} ; 18 \times 22 \mathrm{~cm}-1 \mathrm{~kg} ; 20 \times 32 \mathrm{~cm}$ $-2 \mathrm{~kg}$ ). $\mathrm{O}$ transplantio foi realizado no dia 01/07/97. O substrato utilizado para o enchimento dos sacos plásticos foi o horizonte A de um Latossolo Amarelo, de uma área cultivada com outras espécies, com a seguinte 
composição química: $\mathrm{pH}$ em $\mathrm{H}_{2} \mathrm{O}=5,5$; $\mathrm{Al}=4,9 \% ; \mathrm{K}=10 \mathrm{~g} / \mathrm{kg} ; \mathrm{Ca}=2,9$; $\mathrm{Mg}=0,4 ; \mathrm{P}=6,9 \mathrm{ppm} ; \mathrm{C}=1,6 \%$ ). As plântulas foram avaliadas a cada 30 dias, usando: diâmetro do caule ( $\mathrm{mm}$ ) no colo da planta; altura das plântulas (cm), do colo da planta à última bifurcação de duas folhas; número de folhas abertas. A taxa de crescimento relativo em altura da planta (TCRA) foi calculada por meio da equação TCRA $=\ln A 2-\ln A 1 / t 2-t 1$ (Radford, 1967), em $\mathrm{cm} / \mathrm{cm} / \mathrm{mês}$, onde A1 e A2 são as alturas de duas medidas sucessivas.

As plântulas cultivadas em substrato com esterco tiveram maior crescimento (Tabela 1 ) em relação às sem esterco $(\mathrm{p}<5 \%)$, em todas as avaliações (diâmetro do caule, altura da planta e número de folhas verdes), indicando a vantagem do uso de esterco na formação de mudas para o produtor que deseja levar mudas mais desenvolvidas ao campo em menor tempo. Esta superioridade no crescimento ocasionada pelo esterco confirma as recomendaçôes empiricas de Araujo (1981) e Nishikawa et al. (1998).

Os sacos de $1 \mathrm{~kg}$ e $2 \mathrm{~kg}$ geralmente não diferiram entre si no crescimento das plantas (altura, diâmetro e número de folhas verdes), mas superaram o de $0,5 \mathrm{~kg}$ durante os seis meses de avaliações. Araujo (1991) e Villachica (1996) recomendaram sacos diferentes, dependendo do tempo de viveiro, provavelmente preocupado no crescimento radicular: para mudas com mais de seis meses, eles recomendam sacos com $15 \times 30 \mathrm{~cm}$ $(5,71)$ e para mudas de 4 a 5 meses, recomendaram sacos $10 \times 25 \mathrm{~cm}$ (2 1). Como a semente da pupunha é semeada geralmente no mês de março e as plântulas (com uma folha) estão prontos para transplante no mês de maio, terão em torno de 6 a 7 meses antes de plantio no campo. Portanto, o saco de $1 \mathrm{~kg}(18 \times 22 \mathrm{~cm})$ têm a vantagem sobre o de $2 \mathrm{~kg}$, principalmente no transporte das mudas para o campo, devido ao seu volume, especialmente que o de $2 \mathrm{~kg}$ não dar vantagem no crescimento.

O crescimento de mudas oriundas de plântulas repicadas no estádio de uma folha foi maior $(\mathrm{p}<5 \%)$ que o de plântulas com duas folhas (Tab. 1), mas similar ao de plântulas de chifrinho, em diâmetro e número de folhas. Na altura das plantas, as diferenças foram significativas $(\mathrm{p}<5 \%)$ para os três, com plântulas de uma folha maior, seguida de chifrinhos e de duas folhas.

Em geral, a TCRA nas plântulas com uma folha foi maior do que a média no período entre $30-120$ dias (Fig. 1). A menor TCRA encontrada nos três primeiros meses em plântulas com duas folhas provavelmente foi causada pelo estresse de repicagem e dificuldade de acomodação das raízes (devido ao grande número e comprimento), pois muitas raizes das plântulas podem ser quebradas ou entortadas e levam tempo para adaptarem-se ao novo ambiente emitindo novas raízes. As plântulas 
Tabela 1. Crescimento médio em diâmetro do caule, altura e número de folhas em plântulas de pupunheira, submetidas a diferentes substratos, volumes de substrato e transplantadas em diferentes fases de plântulas, entre agosto de 1997 a janeiro de 1998.

\begin{tabular}{|c|c|c|c|c|c|c|c|c|c|}
\hline \multirow[b]{2}{*}{ Parâmetros } & \multicolumn{3}{|c|}{ Esterco 1} & \multicolumn{3}{|c|}{ Tamanho do saco 1} & \multicolumn{3}{|c|}{ Fases de plântulas1 } \\
\hline & Meses & Com & Sem & $1 / 2 \mathrm{~kg}$ & $1 \mathrm{~kg}$ & $2 \mathrm{~kg}$ & Chifrinho & Uma folha & Duas folhas \\
\hline & ago. & $6,2 \mathrm{a}$ & $5,2 b$ & $5,6 b$ & $6,7 \mathrm{a}$ & $6,1 \mathrm{a}$ & $6,8 \mathrm{a}$ & $7,4 a$ & $4,2 \mathrm{~b}$ \\
\hline Diâmetro & set. & $7,7 \mathrm{a}$ & $6,4 \mathrm{~b}$ & $5,7 b$ & $6,9 a$ & $6,7 a$ & $7,1 \mathrm{a}$ & $7,5 \mathrm{a}$ & $4,6 \mathrm{~b}$ \\
\hline do & out. & $7,6 \mathrm{a}$ & $6,5 b$ & $7,5 \mathrm{~b}$ & $9,6 \mathrm{a}$ & $9,4 \mathrm{a}$ & $9,6 a$ & $10,1 \mathrm{a}$ & $6,7 \mathrm{~b}$ \\
\hline caule & nov. & $12,1 \mathrm{a}$ & $8,6 b$ & $8,7 b$ & $11 \mathrm{a}$ & $11,3 \mathrm{a}$ & $11 \mathrm{a}$ & $11,8 \mathrm{a}$ & $8,2 b$ \\
\hline \multirow[t]{3}{*}{$(\mathrm{mm})$} & dez. & $13,2 \mathrm{a}$ & $8,5 b$ & $8,8 b$ & $11,8 \mathrm{a}$ & $12 a$ & $11,3 \mathrm{a}$ & $11,3 \mathrm{a}$ & $10 \mathrm{~b}$ \\
\hline & jan. & $15 \mathrm{a}$ & $10 \mathrm{~b}$ & $10 \mathrm{~b}$ & $13,3 \mathrm{a}$ & $14,1 \mathrm{a}$ & $12,7 \mathrm{a}$ & $13,2 \mathrm{a}$ & $11,5 b$ \\
\hline & ago. & $6,7 \mathrm{a}$ & $4,5 \mathrm{~b}$ & $5,6 \mathrm{ab}$ & $6 \mathrm{a}$ & $5,2 \mathrm{~b}$ & $6,2 b$ & $6,8 \mathrm{a}$ & $3,7 \mathrm{c}$ \\
\hline Altura & set. & $7,3 a$ & $4,1 \mathrm{~b}$ & $5,3 a$ & $6,1 \mathrm{a}$ & $5,7 a$ & $6 \mathrm{~b}$ & $7,5 \mathrm{a}$ & $3,6 \mathrm{c}$ \\
\hline das & out. & $10 \mathrm{a}$ & $5 b$ & $6,6 \mathrm{~b}$ & $7,9 a$ & $8 a$ & $8,1 b$ & $9,5 \mathrm{a}$ & $4,8 \mathrm{c}$ \\
\hline plantas & nov. & $13,8 \mathrm{a}$ & $6 \mathrm{~b}$ & $8,3 \mathrm{c}$ & $11,2 \mathrm{a}$ & $10,3 b$ & $10,4 b$ & $12,3 \mathrm{a}$ & $6,9 \mathrm{c}$ \\
\hline \multirow[t]{3}{*}{$(\mathrm{cm})$} & dez. & $17,1 \mathrm{a}$ & $7,2 \mathrm{~b}$ & $9,7 b$ & $13,3 \mathrm{a}$ & $13,4 \mathrm{a}$ & $11,9 b$ & $13,7 \mathrm{a}$ & $10,7 \mathrm{c}$ \\
\hline & jan. & $19,2 \mathrm{a}$ & $7,9 \mathrm{~b}$ & $11,1 \mathrm{~b}$ & $14,5 \mathrm{a}$ & $15 a$ & $13,6 \mathrm{~b}$ & $15 a$ & $12,1 \mathrm{c}$ \\
\hline & ago. & $3 a$ & $2,6 b$ & $2,7 a$ & $2,9 a$ & $2,8 \mathrm{a}$ & $3,1 \mathrm{a}$ & $3,2 \mathrm{a}$ & $2,2 b$ \\
\hline Número & set. & $3,6 \mathrm{a}$ & $3,2 \mathrm{~b}$ & $3,1 \mathrm{~b}$ & $3,6 \mathrm{a}$ & $3,4 a b$ & $3,8 \mathrm{a}$ & $3,8 \mathrm{a}$ & $2,6 \mathrm{~b}$ \\
\hline de & out. & $4,4 \mathrm{a}$ & $3,9 \mathrm{~b}$ & $3,8 \mathrm{~b}$ & $4,4 a$ & $4,2 a b$ & $4,5 \mathrm{a}$ & $4,5 \mathrm{a}$ & $3,4 \mathrm{~b}$ \\
\hline folhas & nov. & $4,7 \mathrm{a}$ & $4,1 b$ & $3,9 \mathrm{~b}$ & $4,7 a$ & $4,6 \mathrm{a}$ & $4,6 \mathrm{a}$ & $4,6 \mathrm{a}$ & $3,9 \mathrm{~b}$ \\
\hline \multirow[t]{2}{*}{ verdes } & dez. & $5,1 \mathrm{a}$ & $4,6 \mathrm{~b}$ & $4,3 \mathrm{~b}$ & $5,1 \mathrm{a}$ & $5,2 a$ & $4,7 \mathrm{a}$ & $5 \mathrm{a}$ & $4,9 \mathrm{a}$ \\
\hline & jan. & $5 \mathrm{a}$ & $4,6 b$ & $4,4 \mathrm{~b}$ & $4,9 a$ & $5 \mathrm{a}$ & $4,6 \mathrm{a}$ & $4,8 \mathrm{a}$ & $5 a$ \\
\hline
\end{tabular}

${ }^{1}$ Médias seguidas pelas mesmas letras nas linhas dentro de cada fator em cada data não diferem pelo teste de Tukey a $5 \%$ de probabilidade.

com chifrinhos precisaram de certo tempo para emissão da primeira folha (TCRA negativo no primeiro mês), para dar impulso ao crescimento nos primeiros 60 dias após o transplante. O Villachica (1996) recomenda as 


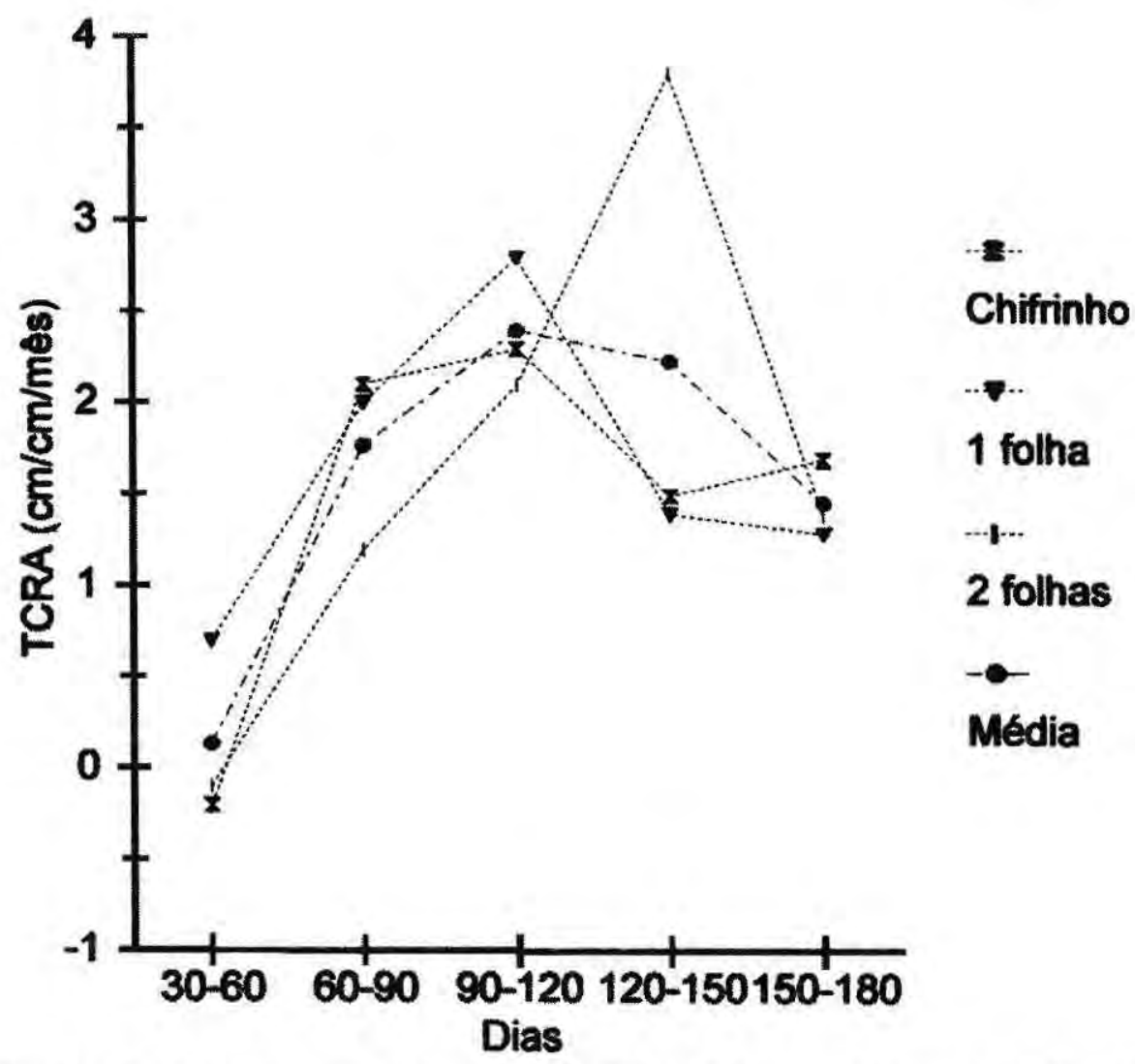

Figura 1. Taxa de crescimento relativo em altura (TCRA) das plântulas de pupunha de 30 a 180 dias após o transplante

plântulas com chifrinhos, que têm facilidade de manipulação e menores possibilidades de quebra das raizes (pouca raiz), e são mais tolerantes ao estresse hídrico em comparação às com folhas abertas. Porém, neste o resultado mostrou que as plântulas com uma folha aberta não mostraram efeito de estresse de transplante e apresentou maior crescimento. Portanto, o melhor estádio das plântulas para a repicagem é a plântula com uma folha, ficando entre a recomendação dada pelo Villachica (1996) de chifrinhos e Nishikawa et al. (1998) de duas folhas abertas.

Concluiu-se que, para a produção de mudas de pupunha de boa qualidade, as plântulas de pupunheira devem ser transplantadas no estádio de uma folha aberta em sacos de um quilo contendo substrato com esterco. Este sistema permite produzir mudas em seis meses e em 
sacos com menor volume de substrato, o que facilita o transporte das mudas para o local definitivo e proporciona mais vantagens aos produtores.

\section{Bibliografia citada}

Araujo, I.C. 1991. Aspectos técnicos da implantação da cultura da pupunheira para produção de palmito. In: Seminário de pupunheira e suas potencialidades econômicas. Anais... Secretaria de Produção Rural e Abastecimento, Manaus. p. 1-38.

CESM. 1996. Normas e padrões para a produção de mudas fiscalizadas. Comissão Estadual de Sementes e Mudas/ DFA/MA/AM, Manaus. 40p.

Clement, C.R.; Mora Urpí, J. 1987. The pejibaye (Bactris gasipaes H.B.K.):
Multi-use potential for the lowland humid tropics. Economic Botany, 41(2):302-311.

Mora Urpi, J.; Vargas, E.; Lopez, C.A.; Villaplana, M.; Allon, G.; Blanco, C. 1982, El pejibaye. Banco Nacional de Costa Rica e Univ. Costa Rica, San José. 16p.

Nishikawa, M.A.N.; Moro, J.R.; Bandel, G. 1998. Cultura da pupunha para produção de palmito. (Série Produtor Rural, $\mathrm{n}^{\circ}$ 6). Universidade de São Paulo/Escola Superior de Agricultura Luiz de Queiroz/Banco Interamericano de Desenvolvimento, Piracicaba. 31p.

Radford, P.J. 1967. Growth analysis formulae - Their use and abuse. Crop Science. 7(3):171-175.

Villachica, H. 1996. Cultivo del pijuayo (Bactris gasipaes Kunth) para palmito em la Amazonia. Tratado de Cooperación Amazonica, Lima. 153p. 